\title{
Erratum: Atom and quantum oscillator coupled by the vacuum field: Radiation pattern, emission spectrum, and decay dynamics [Phys. Rev. A 93, 013804 (2016)]
}

P. R. Berman 1 and C. H. Raymond Ooi

(Q) (Received 16 January 2020; published 31 January 2020)

DOI: 10.1103/PhysRevA.101.019905

Equations (A6a) and (A6b) contain some typographical errors. They should be replaced by

$$
\begin{aligned}
M(\tau) & =-4 e^{-4 \gamma_{a} \tau} T_{0} \frac{r^{2}}{1-r^{2}}+e^{-2 \gamma_{+} \tau}\left[\frac{M_{0}}{2}+\frac{P_{0}}{2}+T_{0} \frac{2 r}{1-r}\right]+e^{-2 \gamma_{-} \tau}\left[\frac{M_{0}}{2}-\frac{P_{0}}{2}-T_{0} \frac{2 r}{1+r}\right], \\
P(\tau) & =-4 e^{-4 \gamma_{a} \tau} T_{0} \frac{r}{1-r^{2}}+e^{-2 \gamma_{+} \tau}\left[\frac{M_{0}}{2}+\frac{P_{0}}{2}+T_{0} \frac{2 r}{1-r}\right]-e^{-2 \gamma_{-} \tau}\left[\frac{M_{0}}{2}-\frac{P_{0}}{2}-T_{0} \frac{2 r}{1+r}\right] .
\end{aligned}
$$

All subsequent equations are correct. 